\title{
Synthetic Research on the Equipment Fault Analysis
}

\author{
Xin REN ${ }^{1,2}$
}

1. Graduate School, National Defense University, Beijing, 100091, China

2. Naval Medical Research Institute, Shanghai, 200433, China

Key words: fault; availability; GO method; Monte Carlo simulation

Abstract: The availability of the equipment system is calculated with the Monte Carlo simulation and GO method based on the principle and operation characteristics of the equipment system in this paper. The analysis results show that this method has high simulation accuracy and can more accurately and objectively reflect the reliability of the equipment system so as to further promote the application and development of the Monte Carlo simulation technology in the reliability analysis of equipment.

\section{Introduction}

GO method is a kind of graphical system reliability modeling and analysis method based on success, which has been applied in the field of weapon equipment system, nuclear power station and pipeline transportation. And Monte Carlo simulation method is a numerical method and it has now been extended to many fields of science and technology, and Monte Carlo simulation technology will play a very important role in the reliability, availability and MTTF evaluation of large complex network system.

\section{GO principle and Monte Carlo method}

GO methodology is a kind of effective system reliability analysis method which is the abbreviation of goal oriented. It is commonly used in multi state and time sequence reliability analysis of the complex system and it is gradually becoming a kind of effective and general analysis method of the system design, reliability and safety analysis.

Monte Carlo, also known as random sampling or statistical test methods, is a method that is used to approximatively solve mathematical problems or physical problems based on the statistical sampling theory. Monte Carlo method belongs to a branch of mathematical calculations and it is a computer simulation method. The basic solving process can be shown as follows in figure 1 .

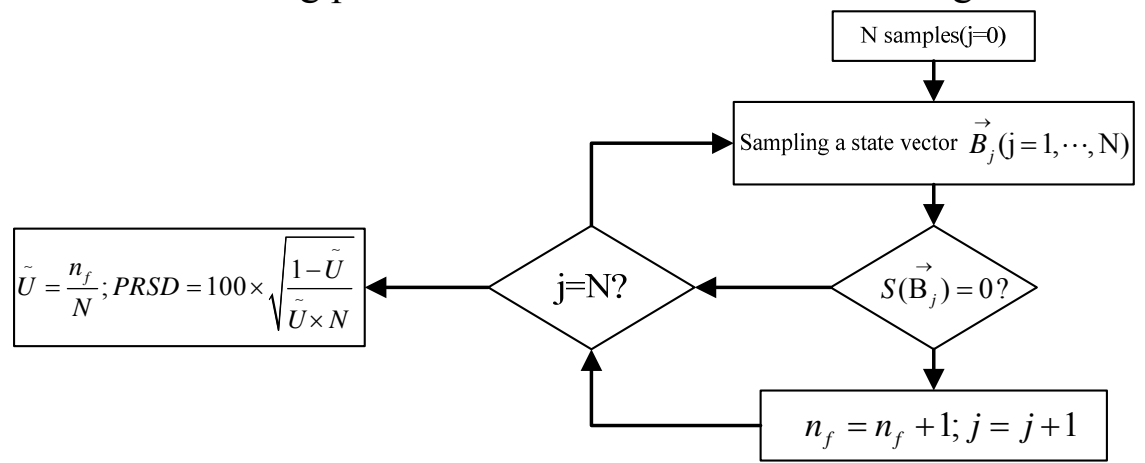

Fig. 1 Flow diagram of Monte Carlo estimation for system availability

Among them, the state space consists of vector $\vec{B}_{j}=\left(b_{1}^{j}, b_{2}^{j}, \cdots, b_{n}^{j}\right)$, the estimated amount is defined as the following random variables $\eta\left(\overrightarrow{B_{j}}\right)=\left\{\begin{array}{cc}1, & S\left(\overrightarrow{B_{j}}\right)=0 \\ 0, & \text { other }\end{array}\right.$, the expected value of $\eta\left(\overrightarrow{B_{j}}\right)$ is as 
follows: $E(\eta)=\sum_{j=1}^{2^{n}} P_{r}\left(\vec{B}_{j}, t\right) \eta\left(\overrightarrow{B_{j}}\right)=\sum_{\substack{j=1 \\ S\left(\vec{B}_{j}\right)=0}}^{2^{n}} P_{r}\left(\vec{B}_{j}, t\right)=U(t)$. Therefore, $\eta\left(\overrightarrow{B_{j}}\right)$ is the unbiased estimator of $U(t)$. The sample of the state vector can be obtained with the application of discrete sampling method, for $i=1, \cdots, n$, to compare the random number $\xi_{\mathrm{i}}$ with $\mathrm{p}_{\mathrm{i}}$, if $\xi_{\mathrm{i}} \leq \mathrm{p}_{\mathrm{i}}(\mathrm{t}), \quad \mathrm{b}_{\mathrm{i}}=1$, otherwise, $\mathrm{b}_{\mathrm{i}}=0$. Now, $\mathrm{U}(\mathrm{T})$ is estimated by using $\mathrm{N}$ history samples:

(1) using $\mathrm{N}$ historical samples, $j=1, \cdots, N$ : a state vector $\overrightarrow{B_{j}}$ is obtained in each historical sample; if $S\left(\overrightarrow{B_{j}}\right)=0, \eta\left(\overrightarrow{B_{j}}\right)=1$, otherwise $\eta\left(\overrightarrow{B_{j}}\right)=0$.

(2) after the calculation of $\mathrm{N}$ historical samples, the estimation of the unavailability is: $\tilde{U}=\frac{1}{N} \sum_{j=1}^{N} \eta\left(\vec{B}_{j}\right)$, the relative standard deviation is: $\varepsilon=100 \times \sqrt{\frac{1-\tilde{U}}{N \times \tilde{U}}}$.

\section{Application example}

\subsection{Equipment system modeling}

The equipment cooling water system is composed of the pumps, heat exchangers and the valves, and it can supply the cooling water to all the equipment which needs to be cooled. The principle diagram of the equipment cooling water system can be shown as follows in figure 2 .

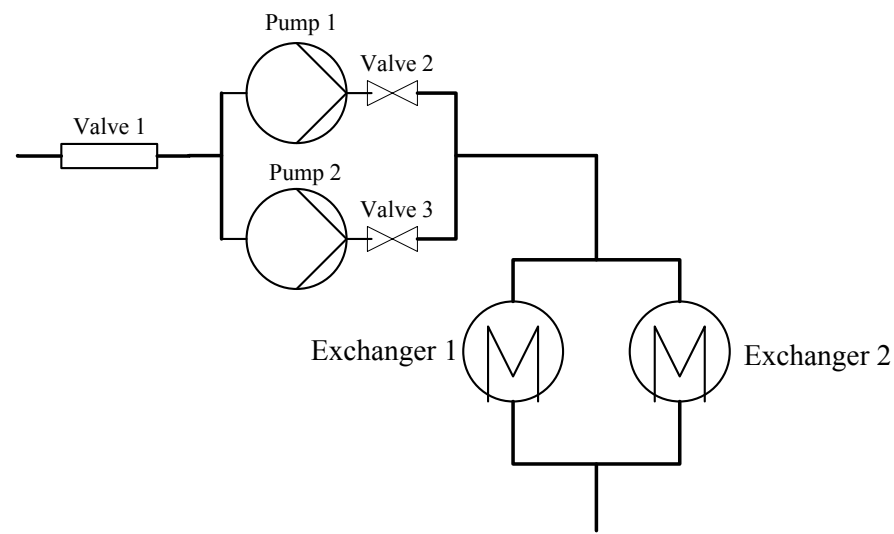

Fig.2 Principle diagram of equipment cooling water system

The unit data of the equipment cooling water system should use the recommended values of International Atomic Energy Agency, as shown in table 1.

Table 1 Data of operators of equipment cooling water system

\begin{tabular}{|c|c|c|c|}
\hline Number & Name of component & Name of fault $/ \mathrm{a}^{-1}$ & Mean maintaince time $/ \mathrm{h}$ \\
\hline 1 & Valve 1 & 5.198 & 3.07 \\
\hline 2,3 & pump 1 and pump 2 & 120.708 & 5.16 \\
\hline 4,5 & Valve 2 and valve 3 & 2.492 & 3.08 \\
\hline 6,7 & $\begin{array}{c}\text { Exchanger 1 and } \\
\text { exchanger 2 }\end{array}$ & 0.594 & 3.05 \\
\hline
\end{tabular}

\subsection{GO method modeling and Monte Carlo simulation analysis}

(1) GO method modeling and analysis

The equipment cooling water system diagram can be established by encoding the operator, and the signal flow, as shown in figure 3 . The water resource and all kinds of control signal is the input signal which can be represented with signal generator operator of type 5, all kinds of valves and heat exchangers can be represented with two state unit of type 1, the control signal of equipment cooling 
water pump can be represented with operator of type 6 and the logic relations can be represented with operator of type 18 .

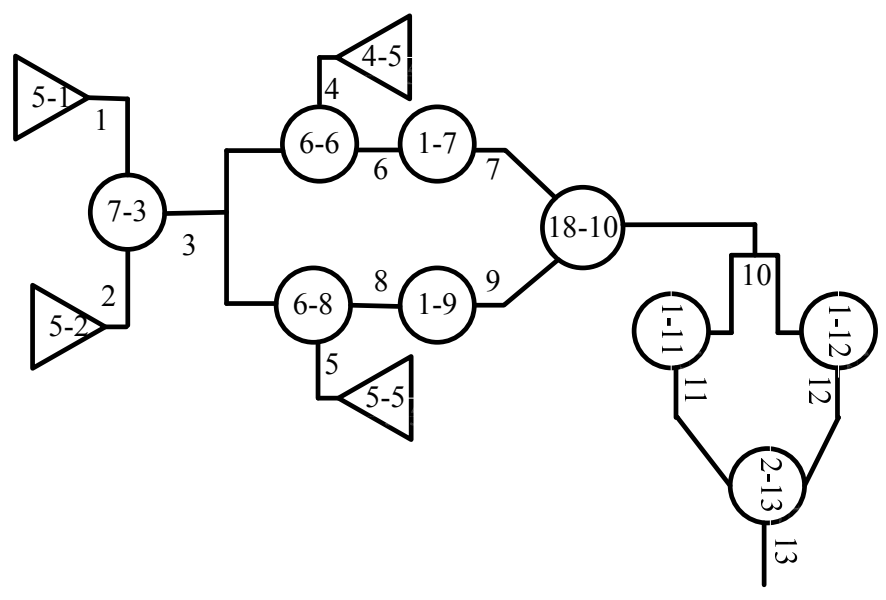

Fig. 3 Go diagram of equipment cooling water system

The unit data of the equipment cooling water system is also recommended by the International Atomic Energy Agency, as shown in table 1. The failure probability of the equipment cooling water system can be calculated within 12 hours, the first step is to calculate the success probabilities of all components in the system at each time point, and it is assumed that the failure rate of the equipment cooling water pump is $\lambda_{\mathrm{i}}$, the repair rate is $\mu_{\mathrm{i}}$, the start failure probability is $\gamma$, and the standby failure rate $\lambda_{\mathrm{jj}}=0, \gamma=0.0236$. The life of other repairable component obeys index distribution, and the failure rate is $\lambda_{\mathrm{j}}$, the repair rate is $\mu_{\mathrm{j}}$. The failure probability of the equipment cooling pumps and other components can be respectively calculated by the two formuls as follows:

$$
\begin{aligned}
Q_{i(t)} & =\frac{\lambda^{*}}{\lambda^{*}+\mu^{*}}+\left(\gamma-\frac{\lambda^{*}}{\lambda^{*}+\mu^{*}}\right) e^{-\left(\lambda^{*}+\mu^{*}\right) t} \\
Q_{j(t)} & =\frac{\lambda_{j}}{\lambda_{j}+\mu_{j}}\left(1-e^{-\left(\lambda_{j}+\mu_{j}\right) t}\right)
\end{aligned}
$$

After the analysis and calculation, the probability of system failure probability can be shown in figure 4.

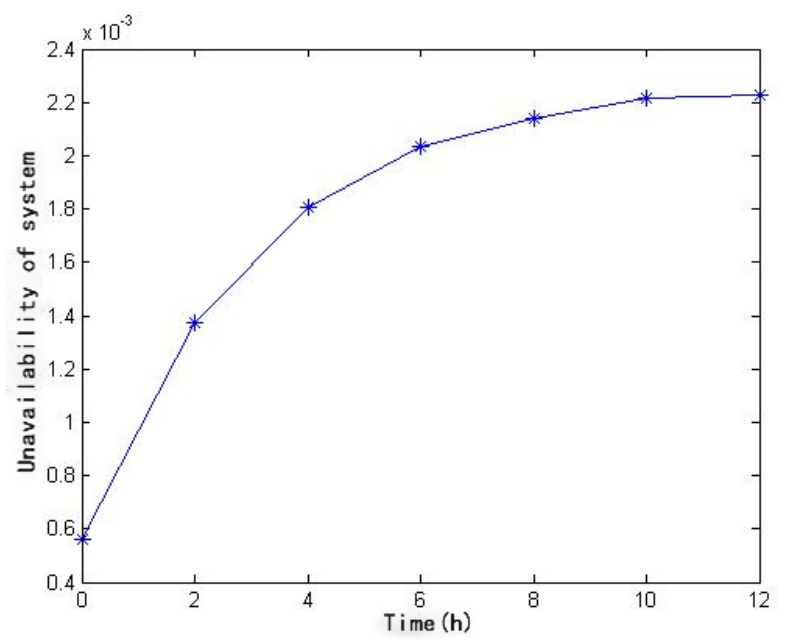

Fig.4 The failure rate tendency diagram of the equipment cooling water system

(2) Monte Carlo simulation analysis

The calculation results of Go method should be compared with Monte Carlo method, and the availability of the equipment cooling water system within 12 hours using Monte Carlo program can 
be shown in figure 5. The solid line in the figure shows the changes of the equipment cooling water system availability along with the time, and the two dotted lines are the envelopes for considering the confidence interval.

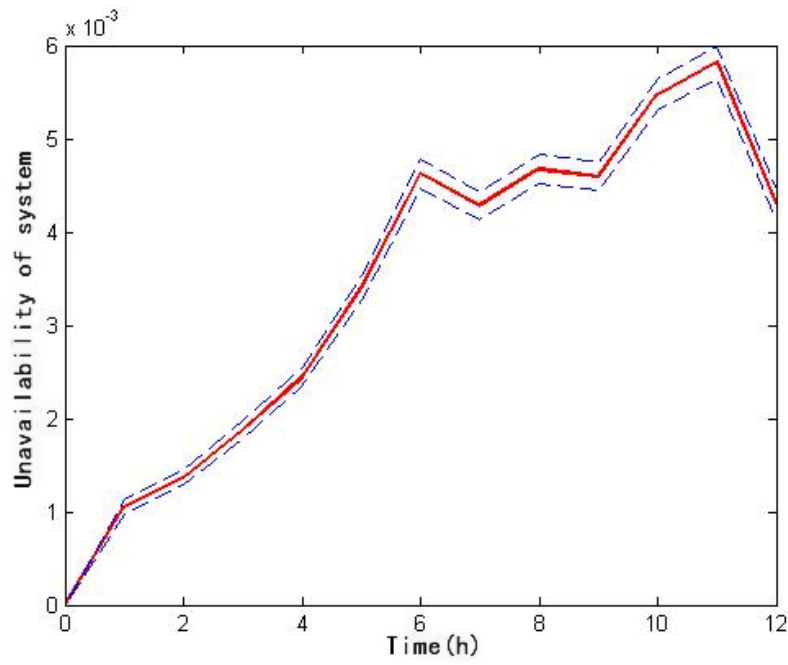

Fig.5 The availability of equipment cooling water system

\section{Conclusion}

The basic principle and solution process of Monte Carlo method are introduced in this paper, and the application of Monte Carlo method in the equipment reliability analysis is discussed. The technical advantage of Monte Carlo method has been fully presented in the equipment reliability analysis and it has been compared with the GO method, and the the availability and standard variance percentage.of equipment system are obtained. The accuracy of calculation results based on Monte Carlo simulation method is very high, which has an important guiding significance on the reliability analysis of equipment system.

\section{References}

[1] International Atomic Energy Agency. Component Reliability Data for Use in Probabilistic Safety Assessment[R]. IAEA-TECDOC-478, 1988, English. 15 Euro.

[2] Retterath B,Venkata S S,Chowdhury A A.Impact of time-varying failure rates on distribution reliability [J].Electrical Power and Energy Systems(S0142-0615),2005,27(9):682:688.

[3] WANG Hongzhou,PHAN Hong.Survey of reliability and availability evaluation of complex networks using monte carlo techniques[J].Microelectron Reliab,1997,37(2):187-209. 